\title{
The Unsupervised Learning Algorithm for Detecting Ellipsoid Objects
}

\author{
Artem V. Kruglov
}

\begin{abstract}
This paper is devoted to the analysis and implementation of the algorithms for automatic detection of the circular objects in the image. The practical aim of this task is development of the algorithm for automatic detection of log abuts in the images of roundwood batches. Based on literature review four methods were chosen for the further analysis and the best performance out of them was provided by ELSD algorithm. Some modifications were implemented to the algorithm to fulfill the requirements of the given task. After all, the modified ELSD algorithm was tested on the dataset of the images. The relative accuracy of the algorithm in comparison with manual measurement is $95.2 \%$ for the images with total area of background scene less than $20 \%$.
\end{abstract}

Index Terms-Arcs grouping, DBSCAN, ELSD algorithm, equivalent circle, hough transform.

\section{INTRODUCTION}

The detection of the log abuts involves the recognition of the objects with radial symmetry on the image. In this regard, we have analyzed the existing algorithms for detecting such objects to identify a suitable method for solving our problem.

One of the most common deterministic methods is the Hough transform (HT) method. On the basis of this method, the algorithm of probabilistic pair voting (PPV) for fast and reliable detection of circular objects is proposed [1]. The algorithm guarantees reliable recognition for overlaps, noise and moderate deformations of the shape. In work [2] an algorithm for recognizing incomplete ellipses based on iterative randomized Hough transform (IRHT) is considered. This method is resistant to strong noise, but it has a high cost of computation and works with objects of a strictly elliptical shape. The authors of the paper [3] developed an algorithm for the localized Hough transform to analyze the Cherenkov radiation, which significantly reduce the time costs for performing Hough transform. In [4], Hough transform is used to estimate the parameters after determining the assumed ellipses by the arc selection strategy.

Other methods for recognizing ellipses include the static RANSAC method described in [5]. In [6], the authors proposed a new scheme for recognizing ellipses using curve segments. A similar algorithm was proposed in [7], where the detection of ellipses is implemented by combining the edge contours according to curvature and convexity.

The method proposed in [8] is a randomized iterative

Manuscript received May 11, 2018; revised April 15, 2019.

A. V. Kruglov is with the Ural Federal University, Yekaterinburg, Russia, 620004 (e-mail: avkruglov@yandex.ru). workflow that uses the geometric properties of the isophot curve in the image to select the most significant edge pixels and classify them into subsets with an equal curvature. In [9], the BFOA algorithm was considered using the example of circle recognition, and its modification was proposed for recognizing a set of figures in the image.

In [10] authors considered algorithms for tracing automobile wheels for the task of constructing an automatic classifier of vehicles. In this paper, a modified algorithm of Viola and Jones is proposed with the construction of a basic feature set over vector gradient map. The method provides good resistance to various lighting conditions and noise with no type II errors.

Good results of recognition of elementary geometric figures is provided by ELSD algorithm [11]. This algorithm effectively detects elliptical arcs and also excludes type II errors.

The most promising methods were tested on the image set of roundwood batches containing 68 images. The test results are shown in Fig. 1.

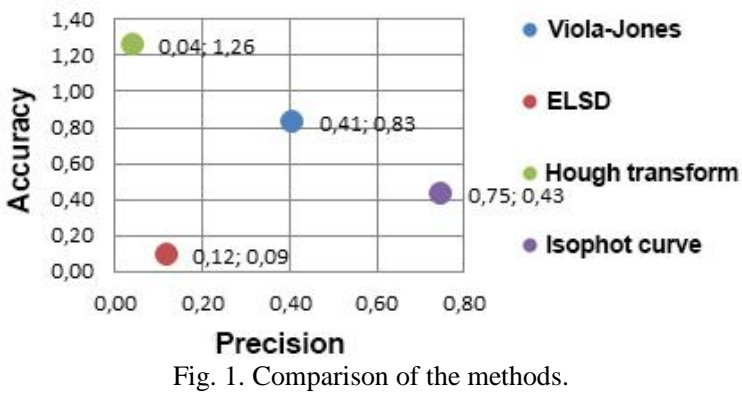

As can be seen from Fig. 1, the best ratio of accuracy and precision is demonstrated by the ELSD method. As a result, this algorithm was chosen for further modification and adaptation.

\section{MATERIALS AND METHODS}

The ELSD algorithm described in [11] is a three-step process:

1) At the first stage, candidates for the desired figures are determined by the heuristic method. The process of region growing from the initial pixel is used at this stage for the segments. The essence of the process lies in the recursive union of pixels with the same gradient orientation (with a given accuracy) into connected regions. For round and elliptical arcs, the curve growing process occurs, which is the second level of grouping: alternating operations of growing the area and forming a chain of regions.

2) Then each candidate passes the validation phase. A 
candidate is any area pixels that could form the desired geometric figure. The method requires two components: a measurement function that gives each candidate an estimate reflecting its rate of structuring, and a model of unstructured data. Candidates will be validated if they are structured as expected in measurement function and meet the model.

The measurement function $k_{x}(c)$ is used to evaluate the structuring of the candidate $c$ in image $x$. It is the number of aligned pixels that the candidate contains. A pixel $p \in \Gamma$ is considered to be aligned with an arc of a circle or ellipse $a$ (see Fig. 2) with an accuracy of $\sigma$ if

$$
\text { Angle }\left(\nabla x(p), \operatorname{dir}_{\perp}\left(\tan _{a}(p)\right)\right) \leq \sigma \pi
$$

where $\nabla x(p)$ is the gradient of the image $x$ at the point $p$, and $\operatorname{dir} r\left(\tan _{\sigma}(p)\right)$ is the direction perpendicular to the tangent $r$ at point $p$.
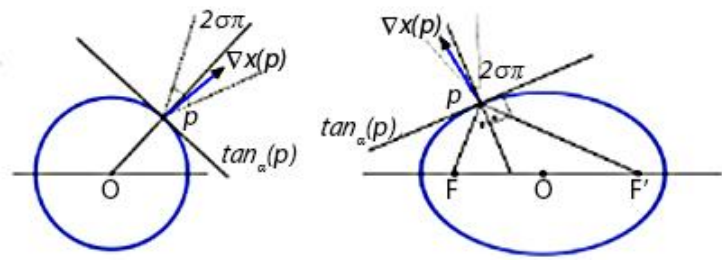

Fig. 2. Pixel alignment.

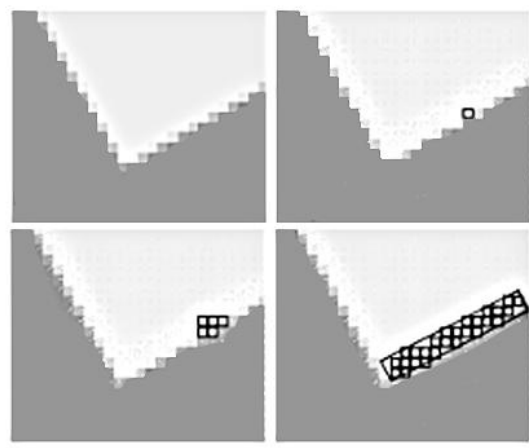

Fig. 3. A principle of region growing.

3) Finally, to select the best geometric interpretation, a model selection step is performed. As a criterion for selecting the model, NFA-values of candidates (Number of False Alarms) are used, that is, the candidate with the minimum value of NFA is considered the most accurate and finally saved in actual detection.

The basics the ELSD algorithm are given in Listing 1.

\section{Listing 1: ELSD}

Input Data: Halftone image $\mathrm{x}$.

Input constraints: None.

Output: $\mathrm{L}_{\mathrm{f}}$ - a list of validated structures (segments, round arcs, elliptical arcs).

grad <- compute_gradient $(x)$;

foreach pixel $p_{i}$ in $x$ do

$R<$-region_grow $\left(p_{i}\right.$, grad $)$;

$C<$ - curve_grow $(R$, grad $)$;

line <-fit_rectangle $(R)$;

circle $<$ - fit_circular_ring $(C)$;

ellipse <-fit elliptical_ring $(C)$;

$\left(N F A_{\text {line }}, N F A_{\text {circle }}, N F A_{\text {ellipse }}\right)=N F A$ (line, circle, ellipse $)$;

$N F A_{\min }<-\min \left(N F A_{\text {line }}, N F A_{\text {circle }}, N F A_{\text {ellipse }}\right)$; if $N F A_{\text {min }} \leq 1$ then

Add the function corresponding to $N F A_{\min }$ to the list $L_{f}$;

end

end

where region_grow is a process of combining neighboring pixels with the same gradient direction into one region (see Fig. 3),

curve_grow is a polygonal approximation, calculation of the area covering the arc (see Fig. 4),

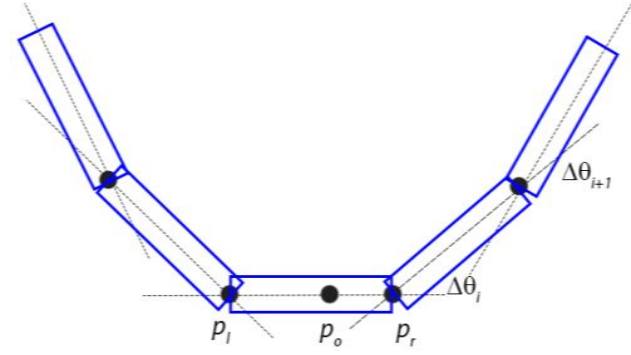

Fig. 4. A principle of curve growing.

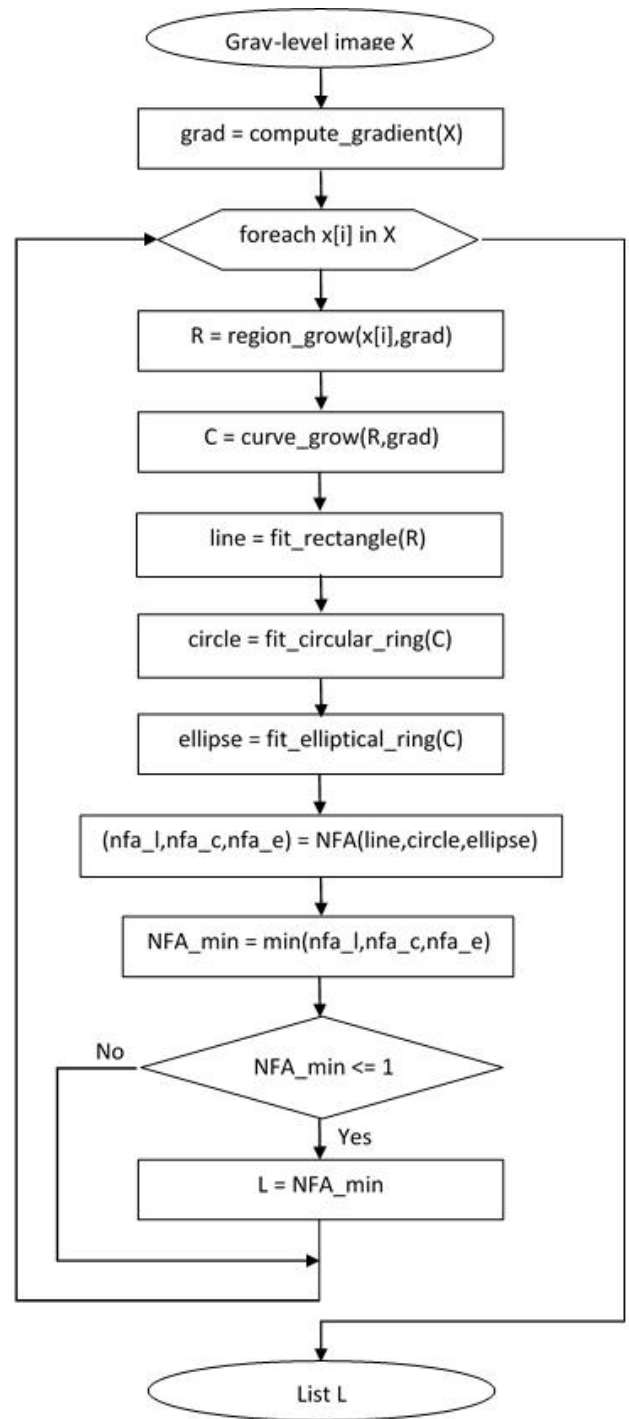

Fig. 5. Control flow graph of ELSD.

Fit_equations is a calculation of equations for constructing circles/ellipses,

Fit_ellipse is an algebraic construction of an ellipse using positional and tangential constraints, 
Fit_circle is an algebraic construction of a circle,

$N F A$ - is a number of false alarms for given candidate.

The, control flow of ELSD algorithm looks like following (see Fig. 5).

Examples of pure ELSD applied to the given problem is given in Fig. 8. As can be seen from these photos, some changes are needed to apply the ELSD algorithm in the log abut detection:

- Discard detection of linear segments which is unnecessary for the given task.

- Discard elliptical and circular arcs with the minimum curvature of the line, since these contours describe linear fragments.

- Discard contours with anomalously minimal and maximum radii after analyzing the radiuses of the arcs. Generally the log abuts in the image have approximately equal radius, and any significant deviation from this range indicates obvious foreign objects in the image.

- Discard segments of curves with small lengths after analyzing the lengths of the arcs as far as these arcs generally belong to the background objects (grass, sawdust).

Major arcs are completed to a full ellipse or circle.
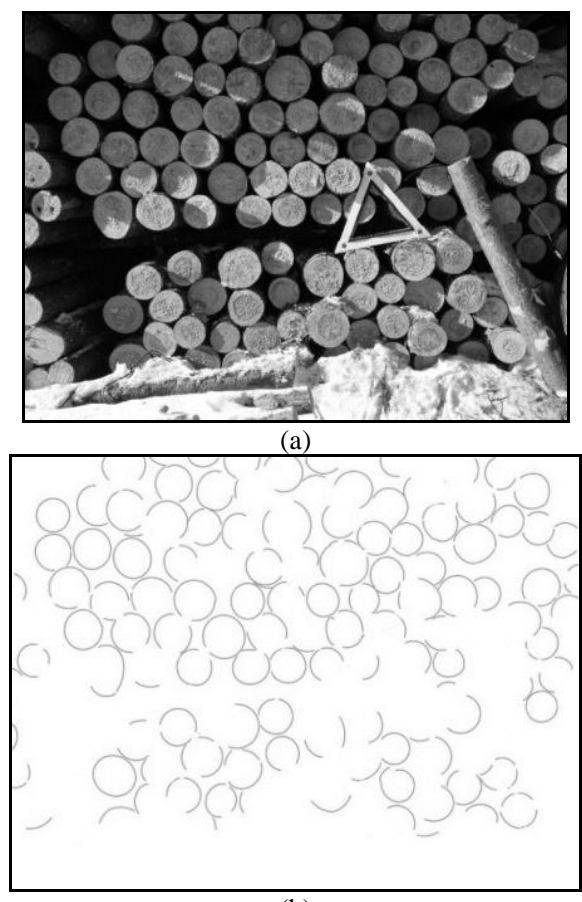

(b)

Fig. 6. Original image (a) and filtered ELSD output (b).

As a result of applying these transformations to the ELSD algorithm, it is possible to obtain an image with unambiguously isolated contours of the ends of the logs, maximally filtered from excessive detection (see Fig. 6(a) and Fig. 6(b)).

To detect the log abut indeed, the original algorithm was supplemented with the following functions:

1) Clustering of the arc centers using the DBSCAN algorithm [12];

2) Determination the center of mass for arc centers;

3) Grouping of the arcs belonging to the same log abut;

4) Approximation by circle the points, belonging to the grouped arcs.
Applying the DBSCAN clustering algorithm, we have received sets of arc centers grouped at the center of the log abut. The next stage is determination the center of mass for each cluster of points. The procedure iteratively adds all the coordinates of the merged points, and then finds the average single point - the candidate of the center of the log abut (see Fig. 7).

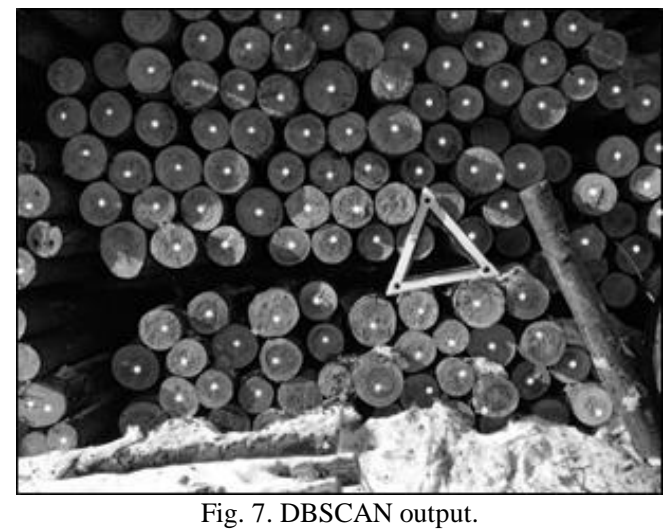

\section{A. Clustering}

The idea of the DBSCAN algorithm is as follows. Let a certain symmetric distance function $\rho(x, y)$ and constants $\varepsilon$ and $\mathrm{m}$ be given. Then

- The domain $E(x)$ for which $\forall y: \rho(x, y) \leq \varepsilon$, is defined as $\varepsilon$-neighborhood of the object $x$.

- A root object or a core object of degree $m$ is an object whose $\varepsilon$-neighborhood contains at least m objects: $|E(x)|$ $\geq m$.

- The object $\mathrm{p}$ is directly reachable from the object $q$, if $p \in$ $E(q)$ and $q$ is a root object.

- The object $\mathrm{p}$ is reachable from the object $q$, if $\exists p 1, p 2 \ldots$ $p n, p 1=q, p n=p$, such that $\forall i \in 1 \ldots n-1: p i+1$ is directly reachable from $p i$.

\section{B. Grouping of the Arcs}

The next step is to allocate the contours obtained after applying the ELSD algorithm to the found centers of log abuts. For this purpose each center of the log abut is associated with the nearest center of the another log abut, so that the approximate radius of selected abut can be determined. Hence near each center point the arcs lying at a distance of the approximate radius and having a convex direction away from the point were found. As a result, it is possible to determine the structure connecting the center point and the arcs belonging to the same log abut [13]:

1) A middle perpendicular $(a, b, c, d)$ is drawn for each arc chord (see Fig. 9).

2) The points of perpendicular intersection are detected.

3) For each pair of arcs, the sums of distances from the middle of the chord to the point of intersection are calculated:

$$
\begin{aligned}
& S a c=A K+C K ; ? \\
& S d c=D L+C L .
\end{aligned}
$$

4) The received sums are sorted in ascending order.

Thus, by setting a certain threshold, we combine arcs with the smallest distances into one structure, which determines the belonging of the arcs to the same log abut. 
a)
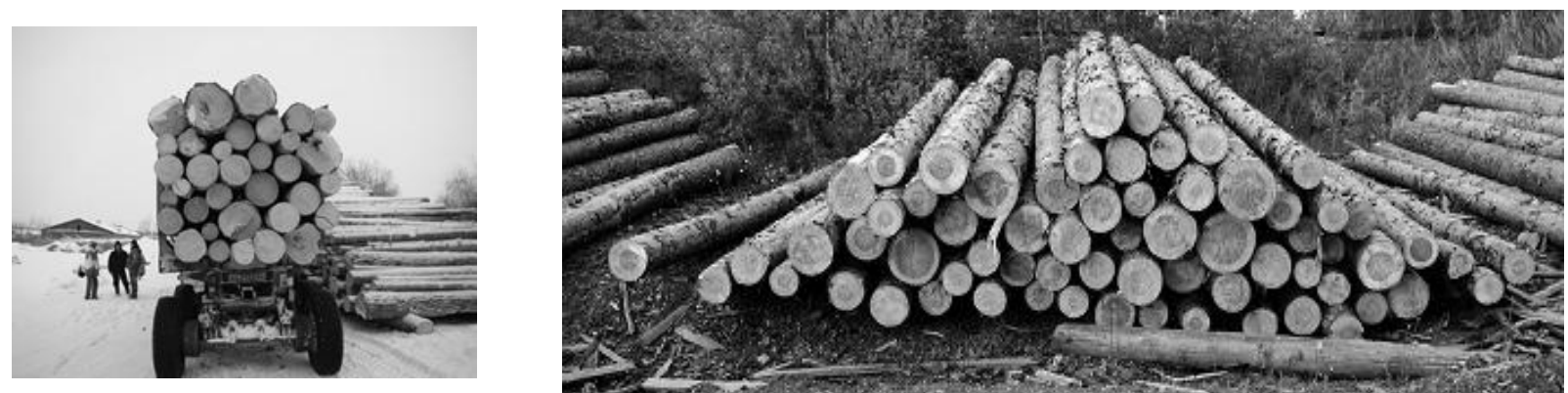

b)
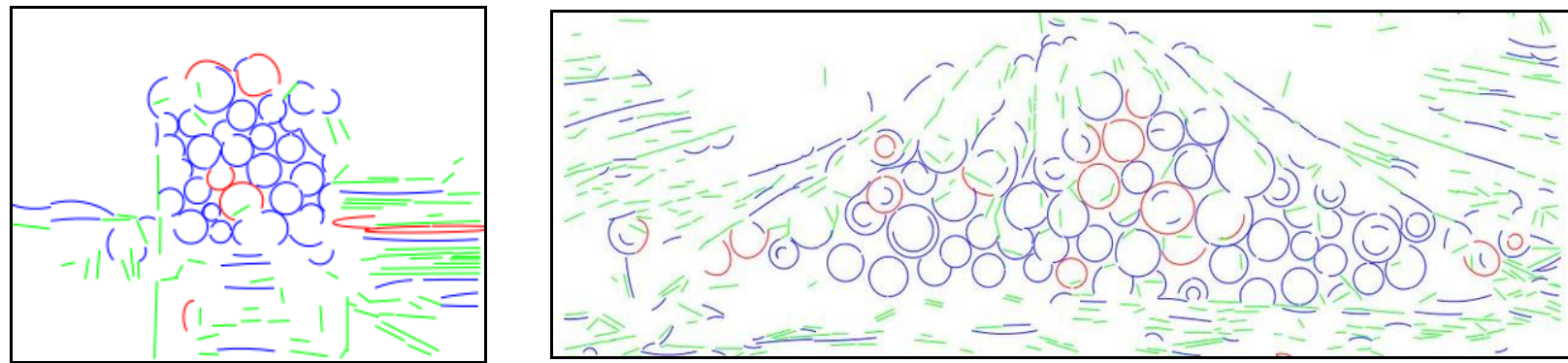

Fig. 8. Original image (a) and original ELSD algorithm output (b).

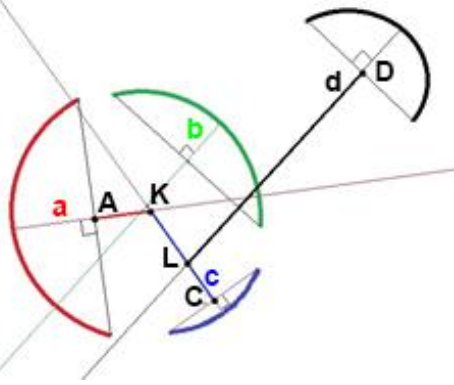

Fig. 9. Chord method.

\section{Approximation by the Equivalent Circle}

After grouping a certain set of points has been obtained, consisting of the center of the log abut and the end points of the arcs, which supposedly describe the target objects of circular shape. To these points the algorithm of approximation by a circle is applied.

In the given task the criterion of algorithmic optimization of OLS [14] is used which is the following:

$$
\begin{gathered}
\min \left\{\sum_{j=1}^{N}\left(\begin{array}{l}
\sqrt{\left(x_{j}-x_{0}\right)^{2}+\left(y_{j}-y_{0}\right)^{2}}- \\
-\frac{1}{N} \sum_{i=1}^{N} \sqrt{\left(x_{i}-x_{0}\right)^{2}+\left(y_{i}-y_{0}\right)^{2}}
\end{array}\right)^{2}\right\} \\
R=\frac{1}{N} \sum_{i=1}^{N} \sqrt{\left(x_{i}-x_{0}\right)^{2}+\left(y_{i}-y_{0}\right)^{2}}
\end{gathered}
$$

where $\left(x_{j}, y_{j}\right)$ - approximated image points $(j=1, \ldots, N)$, $R$-radius of the equivalent approximating circle, $\left(x_{0}, y_{0}\right)-$ center of the circle.

When implementing criterion (1), it is necessary to use nonlinear optimization methods that requires the usage of iterative procedures. Therefore, from the point of view of computational costs, an algebraic approach to approximating a circle is more preferable [13], [15]. In this case, the optimization criterion of OLS is written in the same way as (5), but for squares of distances:

$$
\begin{gathered}
\min \left\{\sum_{j=1}^{N}\left(\begin{array}{c}
\left(x_{j}-x_{0}\right)^{2}+\left(y_{j}-y_{0}\right)^{2}- \\
-\frac{1}{N} \sum_{i=1}^{N}\left(\left(x_{i}-x_{0}\right)^{2}+\left(y_{i}-y_{0}\right)^{2}\right)
\end{array}\right)^{2}\right\} \\
R^{2}=\frac{1}{N} \sum_{i=1}^{N}\left(\left(x_{i}-x_{0}\right)^{2}+\left(y_{i}-y_{0}\right)^{2}\right)
\end{gathered}
$$

In contrast to (3), (4), relation (5) has an analytic solution for the radius (6):

$$
\begin{aligned}
& R^{2}=x_{0}{ }^{2}+y_{0}{ }^{2}+\frac{1}{N} \sum_{i=1}^{N}\left(x_{i}^{2}+y_{i}^{2}\right)- \\
& -\frac{2}{N}\left(x_{0} \sum x_{i}+y_{0} \sum y_{i}\right)
\end{aligned}
$$

Thus, using the found center of the ends, as well as the radii of the approximated circles from the formula (7), one can identify each found object in the image.

As a result of applying these transformations to the original algorithm, we obtain an unambiguously isolated contours of the log abuts, maximally freed from unnecessary detection (see Fig. 10).

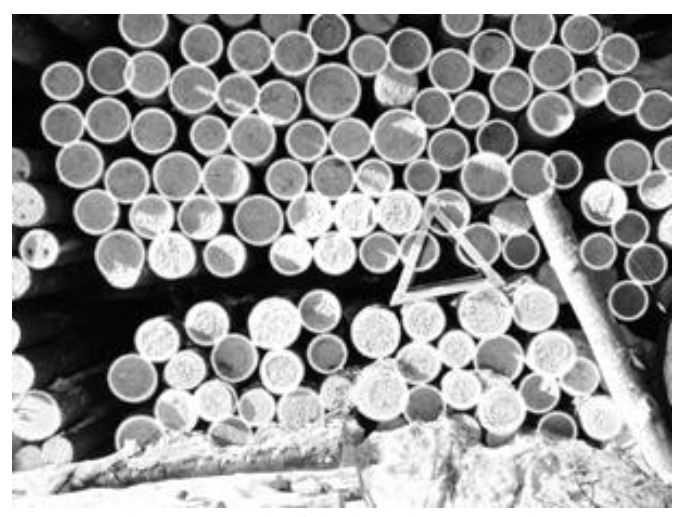

Fig. 10. Result of the $\log$ abuts detection.

\section{TESTING AND RESUlts}

The developed program was tested on the dataset of $\log$ 
batches images, which includes 600 pictures of various $\log$ batches. Testing was conducted on PC Intel Core i5 $2.5 \mathrm{GHz}$; $5 \mathrm{~Gb}$ of RAM.

The result of the testing shows that developed algorithm has a slight dependence on the size of the input image (see Fig. 11).

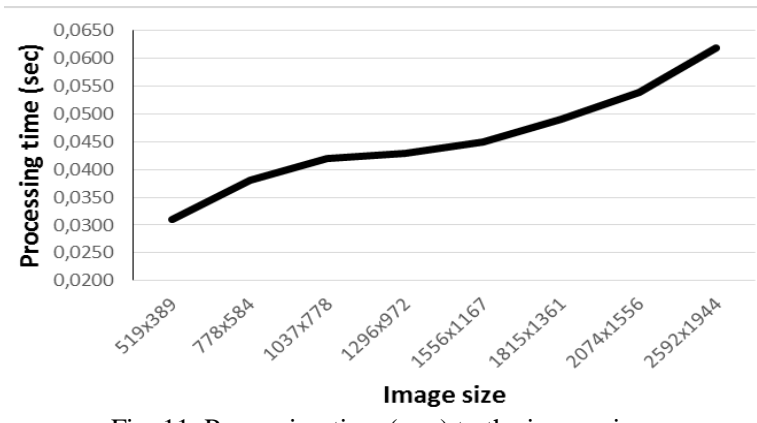

Fig. 11. Processing time (sec.) to the image size.

However, as a result of testing, it was found that the processing time depends exponentially on the number of arcs found in the image (see Fig. 12).

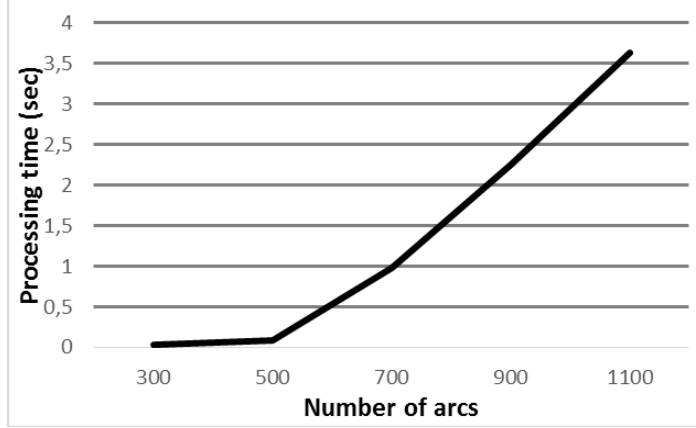

Fig. 12. Processing time (sec.) to the number of arcs.

The result of the testing also shows that the I type errors are typical for images with background objects and can reach up to $30 \%$ (see Fig. 13).

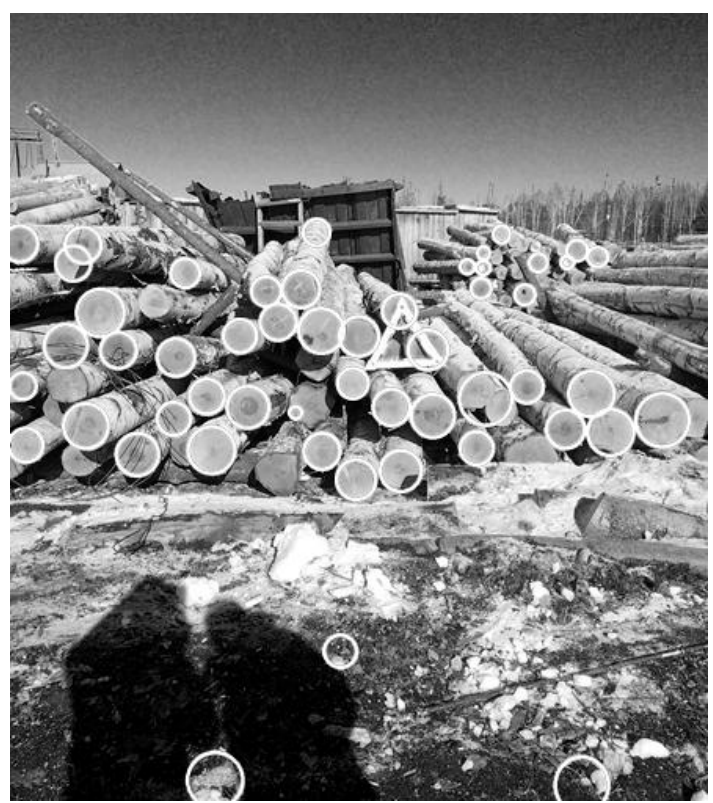

Fig. 13. Image with background objects.

Among the images, the majority area of which is occupied by the target objects, the percentage of false positives tends to zero (see Fig. 14)

The percentage of II type errors ranges from 1 to $7 \%$ depending on the lighting conditions (see Fig. 10). Thus, with the relative area of target objects more than $80 \%$ of the image, the relative accuracy of the algorithm is 0.952 with the minimum accuracy of 0, 929 (see Fig. 15).

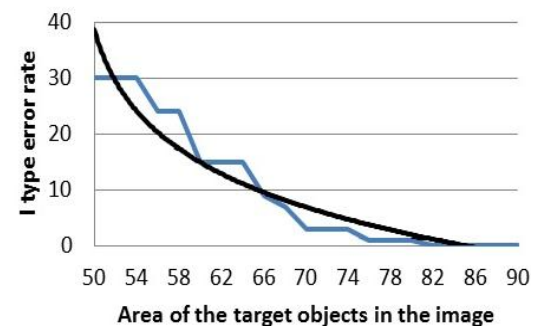

Fig. 14. I type error to the area of target objects.

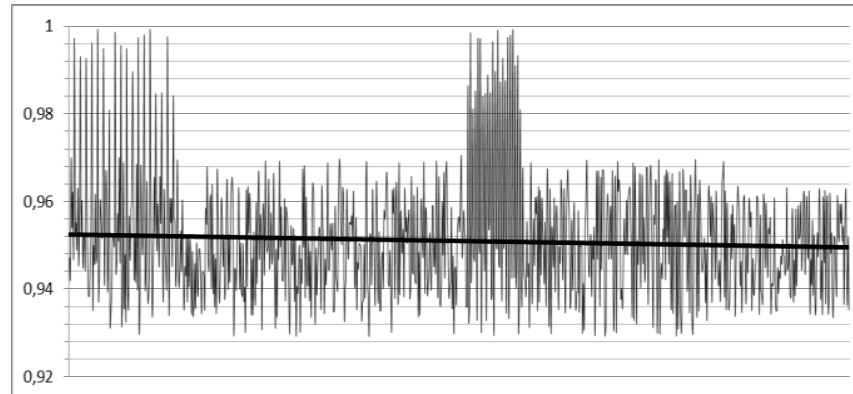

Fig. 15. Relative accuracy of the log abuts automatic detection.

\section{CONCLUSION}

In this paper, we analyzed the applicability of unsupervised algorithms to the problem of detecting the objects of a given type in an image. The study was carried out with reference to the practical task of log abuts detection in roundwood batches. Based on the results of the literature search, 4 algorithms were tested, and as a result, the best performance was provided by the ELSD algorithm described in [11].

The ELSD algorithm was modified to adapt the method to the given task. As a result the algorithm showed good results when detecting objects in images containing a minimal background area. In this case, the accuracy of the algorithm is $95.2 \%$.

Thus, the developed algorithm for detecting objects with radial symmetry can be applied in the timber volume measurement programs.

\section{REFERENCES}

[1] L. Pan, W.-S. Chu, J. M. Saragih, and F. Torre, "Fast and robust circular object detection with probabilistic pairwise voting (PPV)," IEEE Signal Processing Letters, vol. 18, no. 11, pp. 639-642, 2011.

[2] W. Lu and J. Tan, "Detection of incomplete ellipse in images with strong noise by iterative randomized Hough transform (IRHT)," Pattern Recognition, vol. 41, no. 4, pp. 1268-1279, 2008.

[3] S. A. Lebedev and G. A. Ososkov, "Fast algorithms for ring recognition and electron identification in the CBM rich detector," Physics of Particles and Nuclei Letters, vol. 6, no. 2, pp. 161-176, 2009.

[4] M. Fornaciari, A. Prati, and R. Cucchiara, "A fast and effective ellipse detector for embedded vision applications," Pattern Recognition, vol. 47, no. 11, pp. 3693-3708, 2014.

[5] F. Mai, Y. S. Hung, H. Zhong, and W. F. Sze, "A hierarchical approach for fast and robust ellipse extraction," Pattern Recognition, vol. 41, no. 8, pp. 2512-2524, 2008.

[6] K. Hahn, S. Jung, Y. Han, and H. Hahn, "A new algorithm for ellipse detection by curve segments," Pattern Recognition Letters, vol. 13, pp. 1836-1841, 2008. 
[7] D. K. Prasad, M. K. H. Leung, and S.-Y. Cho, "Edge curvature and convexity based ellipse detection method," Pattern Recognition, vol. 45, no. 9, pp. 3204-3221, 2012.

[8] T Marco, D. Cazzato, M. Leo, C. Distante, "Randomized circle detection with isophotes curvature analysis," Pattern Recognition, vol 48, no. 2, pp. 411-421, 2015.

[9] S. M. Mishystin, "Investigation of the BFOA algorithm within the task of recognizing simple figures in an image," in Proc. IV Young Scientist Conf. Information Management Systems and Computer Monitoring, Donetsk, 2013.

[10] S. A. Gladilin, A. A. Kotov, D. P. Nikolaev, and S. A. Usilin, "The construction of stable signs of detection and classification of objects with no characteristic brightness contrasts," Information Technologies and Computer Systems, vol. 1, pp. 53-60, 2014.

[11] V. Patraucean, P. Gurdjos, R. G. von Gioi, "A parameterless line segment and elliptical arc detector with enhanced ellipse fitting," Lecture Notes in Computer Science, vol. 7573, pp. 572-585, 2012.

[12] E. Martin et al., "A density-based algorithm for discovering clusters in large spatial databases with noise," in Proc. the Second International Conference on Knowledge Discovery and Data Mining, AAAI Press, 1996.

[13] U. M. Landau, "Estimation of a circular arc center and its radius," Computer Vision, Graphics and Image Proc, vol. 38, pp. 317-326, 1987.
[14] D. Marquardt, "An algorithm for least squares estimation of nonlinear parameters," SIAM J. Appl. Math., vol. 11, pp. 431-441, 1963.

[15] N. Chernov and C. Lesort, "Least squares fitting of circles," J. of Math Imaging and Vision, vol. 23, pp. 239-251, 2005.

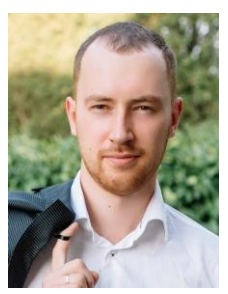

Artem V. Kruglov was born in Yekaterinburg on June 10, 1989. In 2013 he was graduated from Institute of Radioelectronics and Information Technologies, RTF of the Ural Federal University with master's degree in Informatics and counting technics. In 2017 he has defended the PhD thesis in the Ural State Forest Engineering University. Since 2011 he has been involved in a number of research projects related to the industrial control systems and machine vision. Artem Kruglov is an author of 35 papers and 4 patents of Russian Federation. His projects are successfully applied in such industries as metallurgy, mining and forestry. Also he is an organizer of the annual Young Scientists Conference "Information Technologies, Telecommunications and Control Systems". 